\title{
ACIDIFICANTE E DIGESTIBILIDADE DE NUTRIENTES EM SUÍNOS EM CRESCIMENTO
}

\section{ACIDIFIER AND NUTRIENTS DIGESTIBILITY IN GROWING PIGS}

\author{
Juahil Martins Oliveira Jr. ${ }^{*}$ \\ Vanessa Karina Nardi² \\ Antonio João Scandolera ${ }^{2}$ \\ Marson Bruck Warpechowski² \\ ${ }^{1}$ Instituto Federal Catarinense, Araquari, SC, Brasil. \\ 2Universidade Federal do Paraná, Curitiba, PR, Brasil. \\ *Autor para correspondência - juahiljunior@yahoo.com.br
}

\section{Resumo}

Este trabalho teve como objetivo avaliar o efeito da adição de um acidificante em dietas sobre a digestibilidade de nutrientes em suínos em crescimento. Foram utilizados 18 suínos, com peso médio inicial de 53,55 kg, e os tratamentos constituíam de dieta convencional (milho e farelo de soja) e alternativa (milho pré-cozido e soja integral desativada), acrescidas de um acidificante nos níveis 0\%, $0,1 \%$ e $0,2 \%$. Os animais ficaram alojados em três baias coletivas, sendo que em cada arraçoamento eram colocados em boxes individuais. $\mathrm{O}$ método de coleta de fezes foi realizado diretamente na ampola retal e 0,34\% de $\mathrm{Cr}_{2} \mathrm{O}_{3}$ foi utilizado como indicador. $\mathrm{O}$ delineamento experimental utilizado foi de blocos ao acaso, em arranjo fatorial $2 \times 3$ (dietas x níveis de acidificante). A dieta alternativa foi pior para a digestibilidade da $\mathrm{PB}(\mathrm{P}<0,03)$ e resíduo mineral $(\mathrm{P}<0,001)$, porém melhorou a digestibilidade de variáveis da fibra $(\mathrm{FB}, \mathrm{P}<0,001$; e FDA, $\mathrm{P}<0,001)$. A adição de $0,2 \%$ do acidificante às dietas melhorou a digestibilidade do $\mathrm{Ca}(\mathrm{P}<0,01)$. Houve interação entre acidificante e dieta para os coeficientes de digestibilidade da MS $(\mathrm{P}<0,01)$ e FDN $(\mathrm{P}<0,02)$. A dieta alternativa apresentou coeficiente de digestibilidade da MS superior à convencional quando adicionados $0,1 \%$ do acidificante, sendo respectivamente, $86,02 \%$ e $84,51 \%(\mathrm{P}<0,05)$. Nos resultados de digestibilidade da FDN, a dieta alternativa apresentou valor superior à convencional no nível $0 \%$ ou $0,1 \%$ do acidificante. Para a dieta alternativa com $0,2 \%$ foram obtidos valores maiores que nos níveis 0 ou $0,1 \%(\mathrm{P}<0,05)$.

Palavras-chave: Aditivos; milho pré-cozido; óxido de cromo; soja desativada.

\begin{abstract}
The aim of the experiment was to evaluate the effect of the addition of acidifiers to diets on the digestibility of growing pigs. We used 18 pigs with an average initial weight of $53.55 \mathrm{~kg}$. The treatments consisted of conventional diet (corn and soybean meal) and alternative diet (pre-cooked corn and inactive soybean), with the addition of an acidifier at the levels of $0 \%, 0.1 \%$, and $0.2 \%$. The experimental design was in randomized blocks in a $2 \times 3$ factorial arrangement (diets $x$ levels of the acidifier). The alternative diet was worse for $\mathrm{CP}(\mathrm{P}<0.03)$ and ash $(\mathrm{P}<0.001)$ digestibility, but improved fiber digestibility $(\mathrm{CF}, \mathrm{P}$ $<0.001$; ADF, $\mathrm{P}<0.001)$. The addition of the acidifier $(0.2 \%)$ improved digestibility of $\mathrm{Ca}(\mathrm{P}<0.01)$. There was an interaction between the acidifier and diet on digestibility coefficients of DM $(\mathrm{P}<0.01)$ and


$\mathrm{NDF}(\mathrm{P}<0.02)$. The alternative diet showed higher DM digestibility than the conventional diet when added $0.1 \%$ acidifier, respectively, $86.02 \%$ and $84.51 \%(\mathrm{P}<0.05)$. As for NDF digestibility results, the alternative diet showed higher values than the conventional diet with the addition of $0 \%$ or $0.1 \%$ of the acidifier. For the alternative diet with $0.2 \%$, values greater than 0 or $0.1 \%(\mathrm{P}<0.05)$ were obtained.

Keywords: Additives; chrome oxide; pre-cooked corn; inactive soybean.

Recebido em: 15 setembro de 2013

Aceito em: 23 fevereiro de 2017

\section{Introdução}

A produção comercial de suínos foi desenvolvida e vem sendo acompanhada de avanços tecnológicos, buscando melhores índices zootécnicos. Entre os avanços obtidos na área de nutrição animal, o tratamento físico de grãos e a inclusão de aditivos nas dietas visam a melhorias na digestibilidade de nutrientes e redução dos problemas sanitários em suínos. A utilização de antibióticos como promotores de crescimento foi comum nas últimas décadas; no entanto, alguns fatores como a resistência microbiana e os resíduos destes aditivos encontrados em produtos de origem animal contribuíram para sua restrição e desuso em diversos países $^{(1)}$. Dessa forma, a redução na utilização de antibióticos como preventivos na alimentação de suínos trouxe consequências, como a dificuldade de manutenção da sanidade e piora na produtividade, o que impulsionou estudos sobre outros aditivos para as dietas. A adição de acidificantes como aditivos em dietas e seu modo de ação têm sido estudados frequentemente nos últimos anos ${ }^{(2)}$, constituindo-se como uma alternativa promissora que pode contribuir para o aumento da digestibilidade de nutrientes e a redução dos problemas digestivos ${ }^{(3)}$.

As dietas de suínos no Brasil são, normalmente, à base de soja e milho. Estes ingredientes são mais utilizados na alimentação animal por seu elevado teor proteico e energético. No milho, o endosperma representa aproximadamente $83 \%$ do peso seco do grão, consistindo principalmente de amido (88\%), na forma de grânulos altamente organizados, composto por $25 \%$ de amilose e $75 \%$ de amilopectina ${ }^{(4)}$. Este fato pode dificultar a ação de amilases endógenas dos mamíferos e afetar a digestibilidade do amido ${ }^{(5)}$. Já a soja integral apresenta fatores antinutricionais que limitam o seu uso in natura ${ }^{(6)}$, como por exemplo inibidores de tripsina e quimiotripsina, hemaglutininas, fatores bociogênicos, saponinas e lectinas. Nesse contexto, alguns processos como extrusão, micronização e cozimento podem facilitar a ação das enzimas digestivas por promoverem alterações na estrutura química dos nutrientes ${ }^{(5)}$, assim como reduzir a presença dos fatores antinutricionais e, consequentemente, melhorar a digestibilidade dos nutrientes.

Em relação a dietas acidificadas, espera-se que elas possam modular o $\mathrm{pH}$ da digesta do trato gastrointestinal e influenciar os processos fisiológicos da digestão. A inclusão de acidificantes às dietas atua na redução do $\mathrm{pH}$ estomacal e do crescimento de microorganismos indesejáveis, na melhora da digestibilidade dos nutrientes e no aumento da atividade de determinadas enzimas ${ }^{(3)}$. Entretanto, existem muitos resultados controversos em relação à inclusão de acidificantes às dietas e os níveis a serem adicionados às dietas dependem da composição química e propriedades físico-químicas dos alimentos ${ }^{(1)}$. Conforme o exposto, este estudo foi desenvolvido com o objetivo de avaliar o efeito da adição de um acidificante em dietas à base de milho, farelo de soja, milho pré-cozido e soja integral desativada sobre a digestibilidade de nutrientes em suínos em crescimento. 


\section{Material e Métodos}

O experimento foi realizado no Hospital Veterinário da Universidade Federal do Paraná, entre o período de 06 e 26 de outubro de 2007. Foram utilizados 18 suínos (12 fêmeas e 6 machos castrados), com peso inicial e final médio de $53,55 \pm 6,00 \mathrm{~kg}$ e $67,67 \pm 6,78 \mathrm{~kg}$, respectivamente. Os animais foram alojados em três baias coletivas e, no momento dos tratos alimentares, eram alocados em boxes individuais.

O delineamento experimental utilizado foi o bloco casualizado, em arranjo fatorial 2 x 3 (dietas x níveis de acidificante). Os seis tratamentos foram separados em dieta convencional (com milho comum e farelo de soja com $45 \%$ PB) e alternativa (com milho pré-cozido e soja integral desativada), com a adição de acidificante comercial, que continha como princípios ativos 3\% ácido cítrico, $9 \%$ ácido fumárico e $50 \%$ ácido fosfórico, nos níveis de $0 \%, 0,1 \%$ e $0,2 \%$. A composição dos ingredientes e química das dietas convencional e alternativa está apresentada na Tabela 1. Cada animal foi avaliado duas vezes (repetição no tempo), sendo seis repetições por tratamento. A unidade experimental foi o animal, já que cada arraçoamento e coleta de fezes foram realizadas por indivíduo.

As amostras de fezes obtidas de cada animal foram homogeneizadas em amostras únicas (separadas em fase 1 e 2), sendo posteriormente destinadas para a análise de matéria seca (MS), extrato etéreo (EE), fibra bruta (FB), fibra em detergente neutro (FDN), fibra em detergente ácido (FDA), resíduo mineral (RM), matéria orgânica $(\mathrm{MO})$, cálcio $(\mathrm{Ca})$, fósforo $(\mathrm{P})$ e $\mathrm{Cr}_{2} \mathrm{O}_{3}{ }^{(7)}$.

As variáveis experimentais foram os dados dos coeficientes de digestibilidade aparente (\%) de MS (CDMS), EE (CDEE), FB (CDFB), FDN (CDFDN), FDA (CDFDA), RM (CDRM), MO (CDMO), Ca (CDCa) e $\mathrm{P}(\mathrm{CDP})$.

Os animais foram alimentados duas vezes ao dia, sempre às 6:00 e às 18:00. Antes do fornecimento de ração, os animais eram individualizados em boxes de alimentação. Posteriormente, a ração era fornecida e, após 30 minutos, os animais eram liberados e as sobras recolhidas, quantificadas e encaminhadas para a determinação da MS. Nos seis primeiros dias experimentais, foi mensurada a capacidade de ingestão diária máxima de alimento pelo animal. A partir do sétimo dia, foi iniciada a utilização da dieta com inclusão de $0,34 \%$ de óxido de cromo $\left(\mathrm{Cr}_{2} \mathrm{O}_{3}\right)$, considerando-se mais quatro dias para adequação do equilíbrio dinâmico da passagem do indicador. Em seguida, iniciou-se o período de coleta do material experimental, que foi repetida no tempo, dividido em fase 1 e 2 , sendo cinco dias em cada fase. Nesse período, foram realizadas duas coletas diárias de fezes na ampola retal. As amostras foram homogeneizadas todos os dias em cada período, sendo encaminhadas para a pré-secagem à $55^{\circ} \mathrm{C}$.

O milho pré-cozido foi produzido em vapor e pressão $\left(1 \mathrm{kgF} / \mathrm{m}^{2}\right.$ de pressão e $100{ }^{\circ} \mathrm{C}$ de temperatura $)$ durante 15 minutos, seguidos por secagem em secador de fluxo contrário com homogeneizador central. Posteriormente, o milho foi finamente moído em moinho de martelo com peneira de $1 \mathrm{~mm}$. A soja foi processada em vapor e pressão $\left(1 \mathrm{kgF} / \mathrm{m}^{2}\right.$ de pressão e $95^{\circ} \mathrm{C}$ de temperatura) durante 10 minutos. Em seguida, passou por secagem em secador de fluxo contrário com homogeneizador central e moagem em peneira de $2 \mathrm{~mm}$.

Para a avaliação do equilíbrio dinâmico, os dados de concentração de cromo nas fezes foram submetidos à análise de variância para verificação das diferenças entre as fases 1 e 2.

Os dados dos coeficientes de digestibilidade aparente (\%) da MS (CDMS), EE (CDEE), FB (CDFB), 
FDN (CDFDN), FDA (CDFDA), RM (CDRM), MO (CDMO), Ca (CDCa) e P (CDP) foram submetidos ao teste de normalidade de Shapiro-Wilk e à análise de variância a 5\% de probabilidade. Os resultados da análise de variância das variáveis que apresentaram efeito significativo para interação entre dietas e níveis de acidificante foram desdobrados para a análise de significância e teste de comparação de médias pelo teste de Duncan a 5\% de probabilidade. O modelo estatístico utilizado pode ser representado como segue: $Y_{i j k l m}=\mu+\alpha_{i}+\beta_{j}+\tau_{i j}+\gamma_{k(l)}+\varepsilon_{i j k l m}$, sendo $: \mu=$ Média geral dos dados; $\alpha_{i}=$ Efeito do fator dieta; $\beta_{j}=$ Efeito do fator acidificante; $\tau_{i j}=$ Interação dieta $*$ acidificante; $\gamma_{k(l)}=$ Efeito de Fase (Animal); $\varepsilon_{i j k l m}=$ erro aleatório associado a cada observação, $\operatorname{com} \mu=0$ e variância $=\sigma^{2}$.

Tabela 1. Ingredientes e composição química das dietas de farelo de soja e milho moído (Convencional) e de soja integral desativada e milho cozido (Alternativa)

\begin{tabular}{lcc}
\hline Ingredientes & Convencional (\%) & Alternativa (\%) \\
\hline Milho & 66,37 & - \\
Milho Pré-Cozido & - & 62,83 \\
Farelo de Trigo & 3,00 & 3,00 \\
Farelo de Soja & 24,10 & - \\
Soja Integral Desativada & - & 30,40 \\
Óleo de Soja & 2,85 & - \\
Calcário & 1,14 & 1,23 \\
Fosfato Bicálcico & 0,70 & 0,68 \\
Fosfato de Cálcio & 0,57 & 0,57 \\
Sal comum & 0,42 & 0,42 \\
Caolim & 0,29 & 0,29 \\
L-Lisina 78\% & 0,35 & 0,35 \\
DL-Metionina 99\% & 0,09 & 0,13 \\
Mistura Vitamínica & 0,02 & 0,02 \\
Mistura Mineral & 0,10 & 0,10 \\
\hline Composição Química Analisada & & 87,33 \\
\hline \multicolumn{1}{c}{ Matéria Seca \% } & 88,38 & 16,09 \\
Proteína Bruta \% & 16,56 & 8,18 \\
Extrato Etéreo\% & 6,53 & 3,83 \\
Fibra Bruta \% & 3,24 & 24,71 \\
FDN \% & 21,47 & 7,49 \\
FDA \% & 6,92 & 5,00 \\
Resíduo Mineral \% & 5,44 & 0,73 \\
Ca \% & 0,73 & 0,62 \\
$\quad$ P \% & 0,61 & \\
\hline & & \\
\hline
\end{tabular}

\footnotetext{
${ }^{1}$ Mistura Vitamínica (por $\mathrm{kg}$ do produto): nicotinamida: $4.000 \mathrm{mg}$; vitamina A: 1.600 .000 UI; vitamina B12: $3.000 \mathrm{mcg}$; vitamina B6: $442 \mathrm{mg}$; vitamina B2: $400 \mathrm{mg}$; vitamina D3: $320.000 \mathrm{UI}$; vitamina K3: $400 \mathrm{mg}$; vitamina E: $1.600 \mathrm{mg}$; Pantotenato de Ca $90 \mathrm{mg}$; Antioxidante: $2.400 \mathrm{mg}$.

${ }^{2}$ Mistura Mineral (por kg de produto): manganês: $9.400 \mathrm{mg}$; ferro: $14.000 \mathrm{mg}$; zinco: $13.700 \mathrm{mg}$; cobre: 1.800 $\mathrm{mg}$; iodo: $275 \mathrm{mg}$; selênio: $24 \mathrm{mg}$.
} 


\section{Resultados e Discussão}

Não foi verificado efeito da fase $(\mathrm{P}>0,66)$ em relação à concentração de $\mathrm{Cr}_{2} \mathrm{O}_{3}$ nas fezes, o que sugere que o período de quatro dias utilizados para adequação do equilíbrio dinâmico da passagem do indicador foi satisfatório.

Os resultados dos coeficientes de digestibilidade da PB, EE, FB, FDA, RM, MO, Ca e P estão apresentados na Tabela 02. A dieta convencional apresentou valor de 82,15\% para CDPB, sendo este superior $(\mathrm{P}<0,03)$ ao obtido para a dieta alternativa, que foi $79,99 \%$ (Tabela 02). Fan et al. ${ }^{(6)}$ utilizaram leitões fistulados na fase de creche e verificaram que a soja integral extrusada possui maior atividade inibidora da tripsina que o farelo de soja, sendo que as dietas completas com inclusão de soja extrusada pioraram a digestibilidade ileal de alguns aminoácidos (arginina, isoleucina, leucina, fenilalanina, treonina, valina, ácido aspártico, serina e tirosina). Entretanto, os autores citados não evidenciaram diferenças para a digestibilidade aparente da proteína. Já Mendes et al. ${ }^{(8)}$ avaliaram o efeito da inclusão de $25 \%$ de um ingrediente (farelo de soja, soja semi-integral extrusada, soja integral expandida ou soja micronizada) à dieta à base de milho e soja na digestibilidade de nutrientes em suínos em crescimento. Esses autores observaram uma piora nos resultados obtidos do CDPB para dietas com inclusão de soja integral extrusada em até 17 pontos percentuais em relação à dieta com farelo de soja e 21 pontos percentuais em relação à dieta com soja micronizada, o que concorda com os resultados apresentados neste estudo.

O nível de fibra em dietas para suínos pode afetar a digestibilidade de nutrientes ${ }^{(9)}$. Entre as principais propriedades físico-químicas da fibra da dieta relacionadas com efeitos fisiológicos e antinutricionais para monogástricos estão a capacidade de hidratação, a viscosidade, as capacidades de troca catiônica e tamponante ${ }^{(10)}$. Neste estudo, os teores de FB, FDN e FDA foram superiores na dieta alternativa em relação à convencional ( $\mathrm{FB}=3,24 \% ; \quad \mathrm{FDN}=21,47 \% ; \quad \mathrm{FDA}=6,92 \%)$, representando um aumento de 18,21\% para FB, 15,09\% para FDN e 8,24\% para FDA. Esse resultado possivelmente alterou as propriedades físico-químicas da digesta, podendo também ter afetado os valores do CDPB da dieta alternativa em relação à convencional.

A adição de acidificante na ração não afetou $(\mathrm{P}>0,70)$ a digestibilidade da PB (Tabela 02). Entretanto, Machinsky et al. ${ }^{(11)}$, em um experimento com animais em crescimento, verificaram que a inclusão do ácido butírico melhorou a digestibilidade aparente da proteína $(\mathrm{p}<0,05)$. Mroz et al. ${ }^{(12)}$, utilizando suínos fistulados em crescimento, avaliaram o efeito da capacidade tamponante da dieta e/ou a inclusão de ácido fórmico, butírico ou fumárico. Esses autores não obtiveram melhora nos valores de digestibilidade aparente da PB pela inclusão de acidificantes, mas verificaram melhora na digestibilidade da PB nas dietas de baixa capacidade tamponante com a inclusão de ácido fórmico ou butírico. Por outro lado, Blank et al. ${ }^{(13)}$, trabalharam com leitões e dietas à base de farelo de soja e trigo com diferentes capacidades tamponante (pela inclusão de bicarbonato de sódio) e não verificaram efeito da inclusão do ácido fumárico sobre a digestibilidade da proteína. Da mesma forma, Rego et al. ${ }^{(14)}$ trabalharam com leitoas na fase de creche e utilizaram o mesmo acidificante em quantidade semelhante à deste trabalho e também não evidenciaram efeito sobre a digestibilidade da proteína. 
Tabela 2. Coeficientes de digestibilidade aparente (\%) das variáveis e desvio-padrão

\begin{tabular}{|c|c|c|c|c|c|c|c|c|c|}
\hline & \multicolumn{2}{|c|}{ Dietas (D) } & \multirow[t]{2}{*}{ Prob. } & \multicolumn{3}{|c|}{ Nivel de Acidificante (A) } & \multicolumn{2}{|c|}{ Prob } & \multirow[b]{2}{*}{$\mathrm{CV} \%{ }^{4}$} \\
\hline Var $^{1}$ & Conv. $^{2}$ & Alt. $^{3}$ & & $0 \%$ & $0,1 \%$ & $0,2 \%$ & $\mathbf{A}$ & $A^{*} D$ & \\
\hline $\mathrm{PB}$ & $82,15 \pm 2,05^{2}$ & $79,99 \pm 2,70^{b}$ & $<0,03$ & $81,22 \pm 3,10$ & $81,42 \pm 2,27$ & $80,56 \pm 2,53$ & 0,70 & 0,28 & 2,59 \\
\hline $\mathrm{EE}$ & $75,81 \pm 6,83$ & $77,25 \pm 8,43$ & 0,40 & $76,20 \pm 9,47$ & $77,90 \pm 6,29$ & $75,49 \pm 7,14$ & 0,50 & 0,07 & 4,10 \\
\hline $\mathrm{FB}$ & $57,99 \pm 5,31^{b}$ & $67,38 \pm 7,16^{2}$ & $<0,001$ & $64,11 \pm 8,26$ & $63,70 \pm 7,72$ & $60,24 \pm 6,46$ & 0,26 & 0,06 & 5,97 \\
\hline FDA & $68,94 \pm 3,87$ & $72,52 \pm 4,94$ & 0,06 & $70,62 \pm 5,13$ & $71,44 \pm 5,44$ & $70,14 \pm 3,80$ & 0,81 & 0,14 & 4,93 \\
\hline $\mathrm{RM}$ & $54,21 \pm 6,40^{2}$ & $45,75 \pm 6,97^{b}$ & $<0,001$ & $47,21 \pm 8,01$ & $51,03 \pm 5,98$ & $51,71 \pm 9,17$ & 0,15 & 0,10 & 5,69 \\
\hline MO & $87,65 \pm 1,22$ & $87,83 \pm 1,38$ & 0,63 & $87,69 \pm 1,26$ & $88,20 \pm 0,86$ & $87,32 \pm 1,58$ & 0,16 & 0,38 & 1,04 \\
\hline $\mathrm{Ca}$ & $36,12 \pm 9,20$ & $34,76 \pm 9,15$ & 0,56 & $29,99 \pm 6,37^{b}$ & $36,48 \pm 11,58^{2 b}$ & $39,85 \pm 9,70^{2}$ & $<0,01$ & 0,07 & 6,85 \\
\hline $\mathrm{P}$ & $49,72 \pm 10,31$ & $47,59 \pm 7,26$ & 0,35 & $46,47 \pm 6,72$ & $51,18 \pm 8,77$ & $48,33 \pm 9,05$ & 0,25 & 0,36 & 6,59 \\
\hline
\end{tabular}

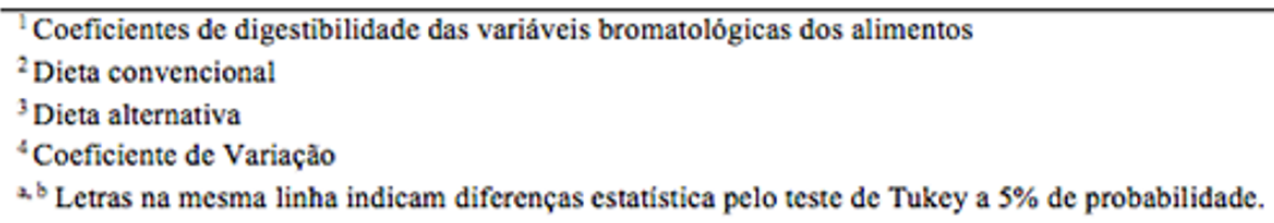

Os resultados obtidos para o CDEE foram $75,81 \%$ para ração convencional e $77,25 \%$ para a alternativa. Em relação aos diferentes níveis de acidificante, os valores obtidos foram 76,20\%, para a dieta sem acidificante, 77,90\%, com adição de 0,1\% de acidificante e 75,49\%, com 0,2\% de adição. Não houve diferença entre o $\operatorname{CDEE}(\mathrm{P}>0,40)$ das diferentes rações como também não houve efeito de acidificante $(\mathrm{p}>0,50)$ ou interação entre acidificante e dieta $(\mathrm{P}>0,07)$. Oliveira et al. ${ }^{(15)}$ também não verificaram diferenças na utilização de milho extrusado em relação ao comum em dietas para leitões na fase inicial. No estudo de Mroz et al. ${ }^{(12)}$, não se verificaram diferenças significativas na digestibilidade aparente do $\mathrm{EE}$ em relação às dietas com diferentes capacidades tamponantes, apenas se evidenciou diferença $(\mathrm{p}<0,05)$ entre dietas quando houve a inclusão de tapioca $(76,6 \%)$, casca de soja $(74,4 \%)$ e polpa de beterraba $(60,9 \%)$. Os resultados do CDEE concordam com os obtidos por Rego et al. ${ }^{(14)}$, que não verificaram diferenças em dietas com a mesma adição do acidificante.

A média obtida de CDFB para a dieta convencional foi menor que a para a alternativa $(\mathrm{P}<0,001)$, sendo observados os valores respectivos de 57,99 e 67,38\%. Já para o CDFDA, os resultados encontrados foram 68,94 e 72,52\%, na mesma ordem, não sendo significativo a 5\% (P>0,06). Sabese que o processo térmico dos grãos e cereais pode alterar a estrutura química dos nutrientes, facilitar a ação das enzimas digestivas e aumentar a digestibilidade de nutrientes ${ }^{(16)}$. Entretanto, na análise de FB, determinam-se celulose e lignina, que são componentes da dieta resistentes à degradação por enzimas endógenas de mamíferos $^{(17)}$. Sendo assim, o tratamento térmico do milho pré-cozido e da soja desativada presentes na dieta alternativa pode ter alterado a estrutura macromolecular da fração fibrosa do alimento, facilitando, assim, a ação e fermentação de microorganismos presentes principalmente no intestino grosso e, consequentemente, ter melhorado a digestibilidade da FB da dieta. Segundo Moreira et al. ${ }^{(5)}$, o processamento do milho e da soja melhoraram a digestibilidade da FB, concordando com os resultados encontrados neste trabalho de que o processamento de grãos pode influenciar a digestibilidade de variáveis da fibra.

Não foi encontrado neste estudo efeito significativo pela inclusão do acidificante na digestibilidade da FB e FDA (CDFB, P>0,26; CDFDA, P>0,81) (Tabela 02). Existe dificuldade em encontrar 
dados publicados sobre a digestibilidade de variáveis da fração fibrosa dos alimentos. Entre as pesquisas avaliando o efeito de acidificantes na digestibilidade em suínos ${ }^{(11-14,18)}$, apenas Rego et al. ${ }^{(14)}$ avaliaram efeito de acidificante sobre a digestibilidade da FDA, sendo os resultados próximos aos encontrados neste estudo (70\%). No entanto, esses autores demonstraram que a dieta com adição de $0,1 \%$ de acidificante apresentou maior CDFDA em relação às dietas com 0 ou $0,2 \%$ $(\mathrm{P}<0,02)$.

Os dados obtidos para CDRM das dietas convencional e alternativa foram, respectivamente, 54,21 e 45,75\%. O CDRM médio da dieta convencional apresentou um valor maior em relação à alternativa $(\mathrm{P}<0,001)$. Da mesma maneira como nas variáveis da fibra, a comparação com outros resultados apresenta o mesmo problema, já que a maioria dos estudos de tratamento térmico de grãos ${ }^{(6,8,15,20)}$ não avaliou a digestibilidade do resíduo mineral. Porém, já foi supracitado que a dieta alternativa apresentou valores de fibra insolúvel (FDN, FDA e FB) consideravelmente superiores aos da dieta convencional pela inclusão principalmente de soja integral. De acordo com Lewis e Southern ${ }^{(9)}$, a fração insolúvel da fibra é considerada um nutriente diluente em dietas para animais monogástricos, sendo relacionada ao aumento no tamanho do bolo fecal, à passagem mais rápida da digesta pelo trato e à piora na digestibilidade. Dessa forma, uma passagem mais rápida da digesta pode ter influenciado negativamente os valores obtidos do CDRM.

Os resultados obtidos para CDRM (Tabela 2) não demonstraram efeito em relação ao nível de acidificante adicionado às dietas $(\mathrm{P}>0,15)$. Na pesquisa de Machinsky et al. ${ }^{(11)}$, não houve efeito da inclusão de ácido butírico às dietas sobre a digestibilidade do resíduo mineral ( $P>0,22)$, assim como Rego et al. ${ }^{(14)}$ também não evidenciaram efeito do acidificante sobre o CDRM. Entretanto, Jongbloed et al. ${ }^{(18)}$ constataram que a inclusão de acidificantes com ou sem fitase às dietas melhorou a digestibilidade do resíduo mineral $(\mathrm{P}<0,001)$, assim como Mroz et al. ${ }^{(12)}$ verificaram que a inclusão de ácido butírico às dietas de alta capacidade tamponante melhorou a digestibilidade do RM.

Nos valores obtidos para CDMO (Tabela 2), não se verificou efeito para dietas $(\mathrm{P}>0,63)$ nem para acidificante $(\mathrm{P}>0,16)$. De acordo com os dados apresentados por Oliveira et al. ${ }^{(15)}$, não houve evidência de que o milho submetido ao processo de extrusão alterou a digestibilidade da MO. Segundo Jongbloed et al. ${ }^{(18)}$, a inclusão de ácido lático ou fórmico melhorou os valores obtidos para CDMO $(\mathrm{P}<0,05)$. Entretanto, Blank et al. ${ }^{(13)}$, Machinsky et al. ${ }^{(11)}$ e Rego et al. ${ }^{(14)}$ não verificaram efeito da inclusão de acidificantes sobre a digestibilidade da MO.

Para o CDCa, não houve diferenças entre as dietas $(\mathrm{P}>0,56)$ nem interação entre dieta e acidificante ( $P>0,07)$; contudo, verificou-se efeito significativo da inclusão de acidificante, sendo que a digestibilidade foi maior $(\mathrm{P}<0,01)$ para dieta com adição de $0,2 \%$ em relação à dieta sem o acidificante $(0 \%=29,99 \%, 0,1 \%=36,48 \%$ e $0,2 \%=39,85 \%)$. Segundo um estudo de Machinsky et al. ${ }^{(11)}$, não houve efeito da inclusão de acidificante sobre o coeficiente de retenção do $\mathrm{Ca}(\mathrm{P}>0,92)$. Entretanto, Jongbloed et al. ${ }^{(18)}$ constataram efeito da inclusão de acidificantes em dietas com ou sem fitase $(\mathrm{P}<0,001)$, que melhorou a digestibilidade do Ca. Uma forma de ação dos acidificantes está relacionada à possibilidade de redução do $\mathrm{pH}$ gástrico e melhor utilização do mineral pelo processo de quelação ${ }^{(3,19)}$. Neste estudo, o efeito significativo do acidificante em relação aos valores obtidos para CDCa pode ser atribuído principalmente a estes fatores, o que pode ter melhorado a solubilidade e consequentemente a digestibilidade do $\mathrm{Ca}$.

Nos resultados obtidos, houve interação entre dieta e acidificante para as variáveis CDMS (p<0,01) 
e CDFDN $(\mathrm{p}<0,02)$, concordando com Suryanarayana et al. ${ }^{(3)}$, que afirmam que o efeito do acidificante é dependente da composição química e dos ingredientes da dieta. O desdobramento das variáveis em que se constatou a interação está apresentado na Tabela 3.

Tabela 3. Coeficientes de digestibilidade aparente das variáveis bromatológicas dos alimentos e desvio-padrão de acordo com a dieta e o nível de acidificante

\begin{tabular}{lccccc}
\hline \multicolumn{7}{c}{ Niveis de Acidificante } \\
\hline Variáveis & Dietas & $\mathbf{0} \%$ & $\mathbf{0 , 1} \%$ & $\mathbf{0 , 2} \%$ & Média \\
\hline \multirow{2}{*}{ MS } & Alternativa & $84,61 \pm 1,78^{\mathrm{Az}}$ & $86,02 \pm 1,15^{\mathrm{Az}}$ & $85,83 \pm 1,78^{\mathrm{Az}}$ & $85,48 \pm 1,63$ \\
& Convencional & $85,87 \pm 1,26^{\mathrm{As}}$ & $84,51 \pm 1,18^{\mathrm{Ab}}$ & $84,42 \pm 1,71^{\wedge z}$ & $84,93 \pm 1,48$ \\
& Média & $85,24 \pm 1,61$ & $85,26 \pm 1,36$ & $85,12 \pm 1,82$ & \\
\multirow{2}{*}{ FDN } & Alternativa & $82,42 \pm 2,83^{\mathrm{Az}}$ & $83,19 \pm 2,50^{\mathrm{As}}$ & $79,22 \pm 2,93^{\mathrm{Ba}}$ & $81,61 \pm 3,14$ \\
& Convencional & $77,29 \pm 2,49^{\wedge \mathrm{b}}$ & $77,10 \pm 2,65^{\wedge \mathrm{b}}$ & $79,67 \pm 5,05^{\wedge z}$ & $78,02 \pm 3,58$ \\
& Média & $79,85 \pm 3,69$ & $80,15 \pm 4,02$ & $79,45 \pm 3,94$ & \\
\hline
\end{tabular}

Variáveis que apresentaram interação entre Dieta ${ }^{\bullet}$ Acidificante (CDMS P<0,01; CDFDN P<0,02).

Coeficiente de variação da análise estatística (CDMS, 1,09\%; CDFDN, 2,49\%).

Letras maiúsculas indicam diferenças significativas na mesma linha e letras minúsculas indicam diferenças significativas na mesma coluna pelo teste de Duncan a $5 \%$.

A dieta alternativa apresentou maior média $(\mathrm{P}<0,05)$ de CDMS que a da dieta convencional quando ambas continham $0,1 \%$ de acidificante, sendo, respectivamente, 86,02\% e 84,51\% (Tabela 3). Já em relação aos diferentes níveis de acidificante adicionados às dietas, não se verificaram diferenças para a dieta convencional nem alternativa. No experimento realizado por Machinsky et al. ${ }^{(11)}$ com animais em crescimento e dietas à base de milho e farelo de soja, não foi verificado nenhum efeito nem interação relacionados ao nível de $\mathrm{Ca}$ da dieta, inclusão de ácido butírico ou fitase sobre a digestibilidade da MS. Entretanto, em trabalho publicado por Jongbloed et al. ${ }^{(18)}$, dietas à base de cevada, farelo de soja, milho, tapioca e farelo de girassol com a inclusão de acidificante (ácido lático ou fórmico) melhoraram a digestibilidade aparente da MS ( $p<0,01)$. Em relação às dietas com inclusão de grãos tratados termicamente, Oliveira et al. ${ }^{(15)}$, em experimento com leitões de 6 a $9 \mathrm{~kg}$, não obtiveram diferenças no CDMS entre dietas com milho comum ou extrusado. Entretanto ${ }^{(8)}$, avaliando o efeito da inclusão de $25 \%$ de um ingrediente (farelo de soja, soja semi-integral extrusada, soja integral expandida ou soja micronizada) à dieta à base de milho e soja sobre a digestibilidade suínos em crescimento, encontraram efeito significativo $(\mathrm{P}<0,05)$ entre todas as dietas, sendo obtido o pior valor de CDMS para a dieta com soja integral extrusada.

Avaliando-se os dados de digestibilidade da FDN, a dieta alternativa apresentou valor superior ao da dieta convencional quando utilizada a adição de $0 \%$ ou $0,1 \%$ de acidificante. Já em relação aos diferentes níveis de acidificante, a dieta alternativa com adição de $0,2 \%$ apresentou valor maior de CDFDN que o nível $0 \%$ ou $0,1 \%(\mathrm{P}<0,05)$. Segundo Suryanarayana et al. ${ }^{(3)}$, os acidificantes podem ter um efeito modulador no $\mathrm{pH}$ da digesta do trato gastrointestinal de suínos. Dessa forma, o acidificante utilizado neste estudo pode ter afetado a fermentação microbiana, influenciando os valores do CDFDN. Em estudo publicado por Rego et al. ${ }^{(14)}$, apesar de os autores trabalharem com animais em fase diferente e não avaliarem a digestibilidade da FDN, a adição de $0,1 \%$ do mesmo acidificante utilizado neste estudo apresentou valores maiores de CDFDA em relação às dietas com $0 \%$ ou $0,2 \%$, sendo os resultados também atribuídos aos efeito do acidificante sobre a modulação de $\mathrm{pH}$ do trato gastrointestinal. 
O principal modo de ação dos acidificantes é pelo efeito antimicrobiano e inibição de patógenos, podendo ainda estimular a secreção de enzimas pancreáticas, reduzir o pH gástrico, agir como fonte de energia durante o metabolismo intermediário do trato gastrointestinal e melhorar a utilização do mineral pelo processo de quelação ${ }^{(3,19)}$. Entretanto, apesar de os autores descreverem esses fatores como premissas para aumentar a digestibilidade aparente total e melhorar o desempenho dos animais, os resultados de estudos utilizando acidificantes ainda são bastante contraditórios. De acordo com revisão publicada por Kill et al. ${ }^{(1)}$, existe uma grande variação nos resultados apresentados em experimentos distintos, sendo que a inconsistência e diferenças encontradas nos trabalhos pode ser devido a variações metodológicas experimentais, principalmente em relação à idade dos animais, composição das dietas e dos acidificantes. Dessa forma, ainda são necessários mais estudos com descrição metodológica detalhada e precisa dos experimentos, para o entendimento mais completo dos principais mecanismos de ação da inclusão ou adição de acidificantes às dietas.

\section{Conclusões}

A adição de acidificante na proporção de $0,2 \%$ melhorou a digestibilidade do cálcio e a adição na mesma proporção para dieta à base de milho pré-cozido e soja integral desativada aumentou a digestibilidade da fibra em detergente neutro.

\section{Referências}

1. Kil DY, Kwon WB, Kim BG. Dietary acidifiers in weanling pig diets: a review. Revista Colombiana de Ciências Pecuárias. 2011,24(3):231-247.

2. Mazzoni M, Gall ML, Filippi SD, Minieri L, Trevisi P, Wolinski J, Lalatta-Costerbosa G, Lallès J, Guilloteau P, Bosi P. Supplemental sodium butyrate stimulates different gastric cells in weaned pigs. The Journal of Nutrition. 2008,138(8):1426-1431.

3. Suryanarayana MVAN, Suresh J, Rajasekhar MV. Organic acids in swine feeding - A review. Agricultural Science research Journals. 2012,2(9):523-533.

4. Paes Maria Cristina Dias. Aspectos físicos, químicos e tecnológicos do grão de milho. Sete Lagoas, MG: Embrapa Milho e Sorgo, 2006.

5. Moreira I, Rostagno HS, Coelho DT. Determinação dos coeficientes de digestibilidade, valores energéticos e índices de controle de qualidade do milho e soja integral processados a calor. Revista Brasileira de Zootecnia. 1994,23(6):916-929.

6. Fan MZ, Sauer WC, Lange CFM. Amino acid digestibility in soybean meal, extruded soybean and full-fat canola for early-weaned pigs. Animal Feed Science and Technology. 1995,52(3-4):189-203.

7. Silva DJ, Queiroz AC. Análise de alimentos: métodos químicos e biológicos. 3.ed. Viçosa: UFV, 2006. $235 \mathrm{p}$. 
8. Mendes WS, Silva IJ, Fontes DO. Composição química e valor nutritivo da soja crua e submetida a diferentes processamentos térmicos para suínos em crescimento. Arquivo Brasileiro de Medicina Veterinária e Zootecnia. 2004,56(2):207-213.

9. Lewis JA., Southern L.L. Swine nutrition. 2th edition, CABI publishing, 2001. 1009 p.

10. Jeraci JL, Van Soest PJ. Improved methods for analysis and biological characterization of fiber. In: New Developments in Dietary Fiber. Springer US, 1990. p. 245-263.

11. Machinsky TG, Kessler AM, Ribeiro AML, Moraes ML, Silva ICM, Cortés MEM. Digestibilidade de nutrientes e balanço de $\mathrm{Ca}$ e $\mathrm{P}$ em suínos recebendo dietas com ácido butírico, fitase e diferentes níveis de cálcio. Ciência Rural. 2010, 40(11):2350-2355.

12. Mroz Z, Jongbloed AW, Partanen KH, Vreman K, Kemme PA, Kogut J. The effects of calcium benzoate in diets with or without organic acids on dietary buffering capacity, apparent digestibility, retention of nutrients, and manure characteristics in swine. Journal of Animal Science. 2000,78(10):2622-2632.

13. Blank R, Mosenthin R, Sauer WC, Huang S. Effect of fumaric acid and dietary buffering capacity on ileal and fecal amino acids digestibilities in early-weaned pigs. Journal of Animal Science. 1999,77(11):2974-2984.

14. Rego JCCR, Ferreira RAS, Brito CF, Moressi GB, Scandolera AJ, Warpechowski MB. Acidificação da dieta e a digestibilidade de nutrientes em leitões. Revista Acadêmica de Ciência Agrárias e Ambientais. 2012,10(1):105-111.

15. Oliveira GC, Moreira I, Furlan AC, Piano LM, Toledo JB, Sierra LMP. Corn types with different nutritional profiles, extrused or not, on piglets (6 to $15 \mathrm{~kg}$ ) feeding. Revista Brasileira de Zootecnia. 2011,40(11):2462-2470.

16. Englyst HN, Cummings JH. Digestion of the polysaccharides of some cereal foods in the human small intestine. The American Journal of Clinical Nutrition. 1985,42(5):778-787.

17. Hall MB. Neutral detergent-soluble carbohydrates, Nutritional relevance and analysis: a laboratory manual. Gainesville: University of Florida, 2000. 42p. (Bulletin, 339).

18. Jongbloed AW, Mroz Z, Weij-Jongbloed VD, Kemme PA. The effects of microbial phytase, organic acids and their interaction in diets for growing pigs. Livestock Production Science. 2000,67(1):113-122.

19. Mroz Z. Organic acids as potential alternatives to antibiotic growth promoters for pigs. Advances in Pork Production. 2005,16:169-182.

20. Moreira I, Oliveira GC, Furlan AC, Patricio VMI., Marcos Junior M. Utilização de farinha prégelatinizada de milho na alimentação de leitões na fase de creche. Digestibilidade e desempenho. Revista Brasileira de Zootecnia. 2001,30(2):440-448. 